\title{
OS TRANSTORNOS DO DESEJO SEXUAL DISORDERS OF SEXUAL DESIRE
}

\author{
Leonardo Gama Filho \\ Pedro Jurberg ${ }^{2}$ \\ Andrea Silva de Moraes $^{3}$
}

\section{Resumo}

0 objetivo deste artigo é a descrição dos novos critérios diagnósticos para os Transtornos de Desejo Sexual, segundo o Manual Diagnóstico e Estatístico de Transtornos Mentais Quarta Edição, Texto Revisado, do ano de 2000, da Associação Americana de Psiquiatria (DSM-IV-TR). Serão discutidos os quadros clínicos, os dados epidemiológicos, os subtipos e os principais diagnósticos diferenciais de cada transtorno. Serão discutidos também os aspectos do "continuum" do desejo sexual, que não se constituem em transtornos, mas em variações normais do desejo. Palavras-chave: Sexualidade - DSM-IV-TR - Transtornos do Desejo Sexual - Critérios Diagnósticos

\section{Abstract}

This article intends the description of news diagnostics criterions to the Disorders of Sexual Desire, from the American Psychiatric Association: Diagnostic and Statistical Manual of Mental Disorders, Fourth Edition,

\footnotetext{
'Mestrando em Sexologia da UGF/RJ, Pós-Graduado em Psiquiatria pela PUC/RJ, Médico do Hospital Municipal Lourenço Jorge/RJ, Médico da Santa Casa de Misericórdia/RJ. Endereço para correspondência: Av. Luiz Carlos Prestes, 410 sala 126 - Barra da Tijuca - Rio de Janeiro (Ed. Barra Trade) Cep: 22.775-055.

E-mail: leonardogamafilho@globo.com

${ }^{2}$ Doutor em Ciências (Neurobiologia) pela UFRJ, Pesquisador Titular do Instituto Oswaldo Cruz - RJ, Professor do Mestrado em Sexologia da UGF - RJ.

E-mail: jurberg@openlink.com.br

${ }^{3}$ Mestranda em Sexologia da UGF/RJ, Psicóloga, Especialista em Psicologia Clinica pela PUC/RJ.
} 
Text Revision, 2000 (DSM-IV-TR). We will discuss clinical scene, epidemiological results, the subtypes and the most important differential diagnostics of each disorder, as well as the aspects of the continuum of sexual desire that does not constitute real disorders, but normal variations of desire.

Key words: Sexuality - DSM-IV-TR - Disorders of Sexual Desire Diagnostic Criterions

\section{Introdução}

0 componente encoberto da sexualidade, ou seja, aquele que ocorre ao nível psicológico e não pode ser observado nem quantificado, é conhecido como desejo ou apetência sexual.

Um dos estudiosos que menciona a importância do desejo sexual, tanto na sexualidade saudável, quanto na etiologia de algumas de suas disfunções, é Helen Kaplan (1977) em um artigo que descrevia, pela primeira vez, a 'Sindrome de Desejo Sexual Hipoativo', conhecida atualmente pelo DSMIV-TR (2000) como 'Transtorno de Desejo Sexual Hipoativo'.

Segundo o DSM-IV-TR (2000), existem dois transtornos na fase de desejo do ciclo de resposta sexual humana: o Transtorno de Desejo Sexual Hipoativo e o Transtorno de Aversão Sexual.

\section{Transtorno do desejo sexual hipoativo}

A caracteristica fundamental do Transtorno de Desejo Sexual Hipoativo é uma "deficiência (ou ausência) persistente ou recorrente de fantasias, ou desejo de ter atividade sexual." (Critério A). Para tal, é necessário que ocorra intenso sofrimento ou dificuldade interpessoal (Critério B). "A disfunção sexual não é mais bem explicada por outro transtorno do Eixo I (exceto uma outra disfunção sexual) nem se deve exclusivamente 
aos efeitos fisiológicos diretos de uma substância (p.ex., droga de abuso, medicamento) ou de uma condição médica geral."(Critério C).

0 estabelecimento de um diagnóstico de Transtorno de Desejo Sexual Hipoativo não é uma tarefa tão simples quanto parece. Quando um individuo queixa-se de redução de libido, deve-se submetê-lo a uma boa entrevista, para avaliar se realmente há uma alteração de desejo sexual. Várias são as circunstâncias possíveis de serem vistas por profissionais que lidam com sexologia e que podem, em um primeiro momento, induzir a um erro de avaliação diagnóstica. Um fato comum é a discrepância de desejo sexual entre parceiros, situação em que o paciente com queixa de falta de desejo sexual pode, na verdade, conviver com uma parceira com um aumento de desejo sexual e que, não necessariamente, devem ser considerados patológicos. Uma diferença importante no desejo sexual, entre os parceiros, é fonte comum de dificuldades sexuais e conjugais (Zilbergeld e Ellison, 1980).

Tipicamente, o parceiro com desejo sexual mais intenso se sente menosprezado e acusa o outro parceiro de disfuncional, enquanto que o parceiro com menor desejo sexual se sente culpado e embaraçado. Um outro problema que também é visto com freqüência, na prática da sexologia, é a incompatibilidade sexual, que é a falta de atração entre os parceiros sexuais e que se constitui em um problema mais grave que a discrepância de desejo sexual.

Kaplan (1995) comenta, a respeito "Algumas pessoas não se sentem atraídas umas pelas outras por boas razões e não faz sentido tentar 'tratar' um indivíduo com um estado de desejo normal baixo, não patológico, como se tivesse um transtorno psiquiátrico."

As causas freqüentes de redução ou bloqueio da atração sexual, devido à incompatibilidade sexual, incluem: feiúra elevada, deformidades, importantes diferenças de idade, retardos mentais, higiene inadequada, comportamentos severamente conturbados (como os encontrados em 
psicóticos), franca incompatibilidade sexual (um homossexual com parceiro do sexo oposto que não o atrai) e uniões que se deram por motivos que não a atração sexual (religiosos, econômicos ou sociais).

Um desejo sexual inibido pode ser o resultado final de uma disfunção primária da fase de excitação sexual, ou da fase de orgasmo, e apresentarse ao clínico como a queixa principal a ser tratada. Neste caso, representa a conseqüência do sofrimento causado pela patologia original e só ocorrerá melhora, em termos de retorno do desejo sexual, quando a disfunção, relativa às fases de excitação ou de orgasmo, for adequadamente diagnosticada e manejada terapeuticamente. Uma outra possibilidade é a de que a inibição do desejo sexual represente a disfunção primária das alterações das fases de orgasmo ou de excitação sexual. No entanto, "alguns individuos com desejo sexual hipoativo retêm a capacidade para a excitação sexual adequada e orgasmo em resposta à estimulação sexual." (DSM-IV-TR, 2000).

\section{Subtipos}

O DSM-IV-TR (2000) oferece subtipos que indicam a natureza do início, do contexto e dos fatores etiológicos envolvidos nas disfunções sexuais. Estes subtipos se aplicam tanto ao Transtorno de Desejo Sexual Hipoativo, como ao Transtorno de Aversão Sexual.

Os subtipos relacionados com a natureza do início da Disfunção Sexual são o 'Tipo Ao Longo da Vida', no caso em que a Disfunção existe desde o começo da atividade sexual, e o 'Tipo Adquirido' quando a Disfunção só se desenvolveu depois de um periodo de funcionamento sexual normal. Os subtipos relacionados ao contexto no qual a Disfunção ocorre são do 'Tipo Generalizado', no caso de a Disfunção Sexual não estar limitada a determinados tipos de estimulação, parceiros ou situações e o 'Tipo Situacional' em que, ao contrário, a Disfunção Sexual fica limitada a estimulações, parceiros ou situações. 
Já os subtipos relacionados com os fatores etiológicos associados à Disfunção Sexual são devidos a "Fatores Psicológicos", quando estes fatores podem ser julgados pelo profissional como exercendo um papel significativo no início, na gravidade, na exacerbação ou na manutenção da Disfunção Sexual, e quando condições médicas gerais ou substâncias não influenciam, de modo algum, na etiologia da Disfunção Sexual; são devidos a "Fatores Combinados", no caso em que tanto os fatores psicológicos como uma condição médica geral ou o uso de substâncias influenciam o inicio, a gravidade, a exacerbação ou a manutenção da Disfunção Sexual. Deve ser dada atenção para o fato de que a condição médica geral ou o uso de substâncias, isoladamente, não são suficientes para explicar a Disfunção. No caso em que uma condição médica geral ou o uso de uma substância for suficiente para explicar a Disfunção Sexual, o diagnóstico a ser feito é o de Disfunção Sexual devida a uma ‘Condição Médica Geral' e/ou Disfunção Sexual 'Induzida por Substância'

Foge, ao objetivo deste trabalho, o detalhamento das condições somáticas que evoluem com a alteração do desejo sexual; porém, devido à alta incidência destas circunstâncias, estas condições serão mencionadas de modo breve.

Toda a patologia que curse com redução do bem-estar do indivíduo e que diminua suas forças e sua auto-estima tem a potencialidade de levar a uma inibição do desejo sexual. Estas doenças também podem alterar a libido de forma direta (por redução de testosterona ou aumento de prolactina, por exemplo). De modo análogo às patologias, há um número muito grande de substâncias que apresentam potencial de inibição do desejo sexual. Algumas substâncias não têm ainda seus mecanismos de ação conhecidos, outras apresentam ação bloqueadora androgênica, outras aumentam os niveis de prolactina, algumas elevam as concentrações de serotonina, reduzindo os niveis de dopamina, e outras ainda atuam de modo indireto, reduzindo o desejo sexual de forma secundária, pois, na verdade, atuam comprometendo as fases de excitação sexual ou de orgasmo. 
O DSM-IV-TR (2000) afirma que um diagnóstico de Transtorno de Desejo Sexual Hipoativo, em geral, não é feito, se o baixo desejo sexual é explicado melhor por um Transtorno Mental do Eixo I (Transtornos Mentais, exceto Retardo Mental ou Transtornos de Personalidade). 0 Manual admite, no entanto, que ambos os diagnósticos podem ser formulados, caso o baixo desejo sexual anteceda o Transtorno do Eixo I, ou quando se apresente como foco de atenção clínica independente.

\section{Epidemologia}

Helen Kaplan (1995) reportou que, dos 5.580 pacientes com Disfunções Sexuais avaliados por sua equipe, no período entre 1972 e 1992, 38\% preenchiam os critérios para Transtornos do Desejo Sexual, apesar de não diferenciar quais os tipos de Transtorno de Desejo e de não fazer referência à incidência entre os sexos. Harold Kaplan e Benjamin Sadock (1995) relataram que o Transtorno de Desejo Sexual Hipoativo é uma queixa mais freqüente nas mulheres e que sua prevalência na população geral é estimada em 20\%.

O DSM-IV-TR (2000) reconhece que existem poucos dados epidemiológicos disponiveis, no que tange às várias Disfunções Sexuais. Os dados citados pelo DSM-IV-TR são oriundos de um estudo realizado nos Estados Unidos (Laumann, Paik e Rosen, 1999), com uma população entre 18 e 59 anos de idade, onde foram inferidas estimativas de prevalências para várias queixas sexuais. No referido estudo, estimou-se que 33\% da população feminina dos Estados Unidos apresentavam Transtorno de Desejo Sexual Hipoativo. Neste estudo, não foi citada a taxa de prevalência para o Transtorno de Desejo Sexual Hipoativo nos homens, nem tampouco a taxa de Transtorno de Aversão sexual para ambos os sexos.

\section{Quadro clínico}

Kaplan (1995) sugeriu um "continuum" para o desejo sexual humano. No ápice deste "continuum”, encontra-se o que ela denominou 'Desejo 
Sexual Hiperativo'. Em seguida, encontra-se o 'Desejo Sexual Normal Alto' e, logo após, o 'Desejo Sexual Normal Baixo'. Continuando esta seqüência, o 'Desejo Sexual Hipoativo Leve' e o 'Desejo Sexual Hipoativo Grave' e, por fim, a ‘Aversão Sexual'. (0 'Desejo Sexual Hiperativo', o 'Desejo Sexual Normal Alto' e a 'Aversão Sexual' serão comentados mais à diante, em tópicos à parte).

Pessoas que apresentam a libido normal, porém regulada em um nivel mais baixo (Desejo Sexual Normal Baixo), tipicamente não procuram ou iniciam espontaneamente atividades sexuais. Contudo, de modo bastante diferente do encontrado nas verdadeiras patologias do desejo sexual inibido, sentem prazer com o sexo, sendo capazes de obter ereções, lubrificações vaginais e excelentes orgasmos. 0 que causa problema neste grupo de pessoas é a freqüência de aproximações sexuais ultrapassar um limite superior ao estabelecido pelo seu padrão individual de baixo desejo sexual. Um impulso sexual, mesmo que considerado normal, pode se tornar problemático, se o desejo sexual do parceiro apresentar-se demasiadamente mais alto.

Contrastando com a variante normal baixa do "continuum" do desejo sexual, no Desejo Sexual Hipoativo Leve não há uma resposta genital satisfatória à estimulação erótica com parceiros adequados. Tipicamente, estes pacientes referem dificuldades de tornarem-se sexualmente excitados, necessitando de intensa estimulação para que possam experimentar um orgasmo. Na maioria das vezes, seus orgasmos são percebidos como uma experiência rápida e transitória, não apresentam fantasias sexuais e freqüentemente passam longos periodos de tempo sem atividades sexuais, fato que não produz desconforto ou frustração aparente.

Apesar da falta de desejo sexual, estes pacientes podem, eventualmente, procurar seus parceiros ativamente para fins sexuais, geralmente para agradá-los. É freqüente que apresentem alterações de lubrificação vaginal, manutenção da ereção e/ou obtenção do orgasmo. Quando conseguem uma atividade 'normal', esta é referida como mecânica ou 
quase que desprovida de prazer. Alguns pacientes relatam que, eventualmente, conseguem obter orgasmos verdadeiramente satisfatórios, mas que logo após ocorre um retorno ao nível anterior de baixo desejo sexual. Sentem-se muito confusos, pois não conseguem entender como desfrutam de uma experiência tão gratificante e depois ficam tão desmotivados para repeti-la.

No Transtorno de Desejo Sexual Hipoativo Grave, o paciente não experimenta qualquer forma de excitação sexual, mesmo que submetido às mais intensas estimulações físicas e/ou psíquicas. Tais pacientes não gostam de sexo e relatam espontaneamente que não sentem a menor falta de atividade sexual em suas vidas. Geralmente levam uma vida celibatária e evitam ao máximo o contato sexual. Kaplan (1995) utilizou o termo "anorexia sexual", para descrever o baixíssimo apetite sexual visto nestes pacientes.

Nos individuos onde raramente ocorre alguma forma de manifestação sexual, esta é referida como sendo neutra, desprovida de prazer ou apenas tolerável. Como tais pessoas encontram-se satisfeitas em ter a atividade sexual fora de suas vidas, é pouco provável que procurem ajuda para seus problemas, somente sendo vistas em consultas especiạlizadas quando sua inibição de desejo sexual resultar em sérios problemas conjugais, ou então quando levar a uma incapacidade de formação de vínculos interpessoais, causando solidão, isolamento e possiveis sentimentos depressivos.

\section{Transtorno de aversão sexual}

Para o DSM-IV-TR, a característica essencial do Transtorno de Aversão Sexual é a intensa aversão ou esquiva, persistente ou recorrente, da maioria dos contatos sexuais genitais com um parceiro sexual (Critério A); a perturbação deve causar grande sofrimento ou dificuldade interpessoal (Critério B). A disfunção não é explicada melhor por outro transtorno do eixo I, exceto por outra Disfunção Sexual (Critério C). 
Este transtorno apresenta-se sob forma de uma fobia de proporções graves, onde o individuo teme o ato sexual, ou a idéia dele. 0 paciente percebe o caráter irracional e desproporcional de seus temores, porém, não é capaz de enfrentar a situação considerada como ameaçadora. Segundo Masters, Johnson \& Kolodny (1994), a Aversão Sexual é de duas a três vezes mais freqüente em mulheres.

Kaplan (1995) considera a Aversão Sexual como sendo a forma mais severa de transtorno do desejo sexual. Alguns individuos apresentam a forma Generalizada do Transtorno, quando ocorrem sentimentos intensos de ansiedade e/ou repulsa a toda e qualquer sensação, sentimento, pensamento, imagem ou oportunidade erótica.

Na sua forma Situacional, a Aversão Sexual encontra-se restrita a determinadas circunstâncias relativas à atividade sexual. Kaplan (1995) lista treze formas mais comuns da Aversão Sexual do tipo Situacional, que são as seguintes: tocar e acariciar o corpo; a visão da genitália (própria ou do parceiro); tocar a genitália do parceiro(a); beijar (principalmente boca, seios e genitais); penetração vaginal (mulheres: sendo penetrada com o pênis ou com qualquer outro objeto; homens: penetrar); secreções e odores sexuais (sêmen, secreções vaginais); excitação sexual (a própria ou a do parceiro); orgasmo (medo da perda do controle); sexo oral (receber ou fazer); falha sexual (forma mais freqüente em homens, medo de desempenho); gravidez (ficar ou causar); contrair uma doença sexualmente transmissível, e nudez (a própria ou a do parceiro(a).

Alguns pacientes com Aversão Sexual do tipo Situacional podem apresentar funcionamento sexual normal, desde que consigam evitar e manter, sob seu controle, as situações fóbicas especificas. 0s pacientes com a forma Generalizada de Aversão Sexual tipicamente demonstram um comprometimento sexual muito mais grave e incapacitante. Na Aversão Sexual do tipo Ao Longo da Vida, uma forma menos comum, o paciente nunca experimentou a experiência sexual, sem que alguma 
forma de manifestação fóbica estivesse presente. Na sua forma Adquirida, ela surge após a ocorrência de uma história de atividade sexual normal prévia.

Assim como em outras fobias, o individuo portador de Aversão Sexual também apresenta comportamentos característicos destes Transtornos de Ansiedade, tais como ansiedade antecipatória, evitação e reação fóbica. 0 paciente experimenta alterações fisiológicas como sudorese, vômitos, náuseas, taquicardia, dispnéia, tonturas, parestesias, desmaios, etc., quando ele pensa ou enfrenta uma situação fóbica, produzindo, respectivamente, ansiedade antecipatória ou reação fóbica. A intensidade do temor é de tal grandeza que pode precipitar um verdadeiro ataque de pânico, no paciente. Uma variedade de comportamentos voltados para a evitação da atividade sexual é encontrada nestes individuos e as estratégias desenvolvidas por eles são engenhosas e incluem atividades intencionalmente voltadas ao impedimento físico $\mathrm{e}$ emocional para o sexo.

Com relação ao diagnóstico diferencial, a principal condição a ser excluida é a Dispareunia, pois qualquer que seja a causa de dor durante o coito, a associação freqüente de sexo e dor pode ocasionar perda de desejo sexual e/ou desenvolvimento de Aversão Sexual. Obviamente, quando detectada uma causa física que explique dor durante a atividade sexual, esta deve ser tratada, geralmente por um profissional ginecologista ou urologista. Nestas circunstâncias, o DSM-IV-TR aconselha que ambas as condições sejam diagnosticadas.

\section{Desejo sexual elevado}

Dentro do continuum do desejo sexual, existem pessoas que apresentam impulsos libidinais muito fortes e intensos, sem que possuam qualquer patologia sexual. Kaplan (1995) denominou-as portadoras de "Desejo Sexual Normal Elevado". Tais indivíduos sentem-se estimulados por uma gama de situações, cenários e parceiros 
sexuais, apresentando oscilações espontâneas em seu desejo sexual. Tipicamente sentem-se satisfeitos com suas atividades sexuais e as têm como altamente gratificantes. 0 que confere a esses individuos um caráter egossintônico, em relação ao seu impulso sexual alto, é que eles conseguem perfeitamente controlá-lo em situações onde o impulso poderia mostrar-se, de alguma forma, inadequado ou com potencial para acarretar algum prejuizo individual (social, econômico, ocupacional, etc). Uma outra característica é a ausência de ansiedade ou qualquer outra queixa referente à sua sexualidade intensa, pois tanto o indivíduo quanto seus parceiros, ao contrário disso, sentem que a elevação do desejo sexual contribui para a melhora de suas vidas e de seus relacionamentos.

0 desejo sexual patologicamente elevado já era conhecido por profissionais de saúde mental, há séculos, e recebeu a denominação de "ninfomania", quando presente em mulheres, e de "don juanismo" ou "satiriase", quando presente em homens. Kaplan (1979) cunha o termo "Desejo Sexual Hiperativo" e o descreve como sendo uma entidade clínica relativamente rara e mais freqüente no sexo masculino. A sua principal característica é a marcante falta de controle sobre a motivação sexual, sendo que os individuos mostram-se insaciáveis sexualmente e o orgasmo não reduz suas necessidades de mais estimulações sexuais. 0 caráter irresistivel da libido, assim como a falta de controle sobre esta, levam-nos a buscar freneticamente novas atividades sexuais, mesmo que estas se mostrem potencialmente nocivas a vários aspectos da suas vidas. Freqüentemente, os pacientes com Desejo Sexual Hiperativo demonstram sofrimento e preocupações sobre as conseqüências que poderiam surgir, decorrentes de suas atividades sexuais intensas e insaciáveis, em relação às suas atividades profissionais, sociais, econômicas, aos seus relacionamentos afetivos e a sua saúde física e mental. Muitas vezes, estas pessoas tentam ativamente abster-se sexualmente, o que na maioria das vezes ocasiona uma sensação de ansiedade intensa e disforia, fazendo com que a grande maioria deles retome às suas atividades sexuais hiperativas. 
0 verdadeiro Transtorno de Desejo Sexual Hiperativo deve ser diferenciado dos estados de hipersexualidade, vistos em quadros de mania ou de hipomania (Transtornos Afetivos) e dos estados de desinibição sexual, encontrados em retardos mentais e nos estágios inicias das demências. 0 Transtorno de Desejo Sexual Hiperativo também é conhecido pelos nomes de "sexualidade compulsiva" e "adição sexual". Tais terminologias são inexatas, pois a hipersexualidade destes individuos não se constitui, no verdadeiro sentido psicopatológico, nem em uma dependência, nem em uma compulsão, sendo mais bem entendida como resultante de uma falha de controle de impulsos que apresentam potencialidade perigosa para o próprio indivíduo ou para terceiros.

0 Transtorno de Desejo Sexual Hiperativo não é considerado pelo DSMIV-TR (2000) como uma entidade nosológica independente, podendo, entretanto, ser classificado como Transtorno Sexual Sem Outra Especificação. Na CID-10 (1992), estes indivíduos são diagnosticados como portadores de Impulso Sexual Excessivo.

\section{Referências bibliográficas}

American Psychiatric Association: Diagnostic and Statistical Manual of Mental Disorders, Fourth Edition, Text Revision. Washington, DC, American Psychiatric Association, 2000.

KAPLAN, H. S. Hipoactive Sexual Desire. J. of Sex and Marital Therapy 3 (1), 1977.

KAPLAN, H. S. O Desejo Sexual. Rio de Janeiro: Nova Fronteira, 1983. KAPLAN, H. S. Transtornos do Desejo Sexual. Porto Alegre: Artmed, 1999. KAPLAN, H. L., SADOCK, B. J. Tratado de Psiquiatria. Porto Alegre: Artmed, 1999.

LAUMANN, E. O., PAIK, A., ROSEN, R.C. Sexual Dysfunction in the United States: Prevalence and Predictors. The Journal of the American Medical Association: 1999 281: 537-544.

MASTERS, W. H., JOHNSON, V.E., KOLODNY, R.C. Heterossexualidade. Rio de Janeiro: Bertrand, 1997. 
WORLD HEALTH ORGANIZATION: The ICD-10 Classification of Mental and Behavioural Disorders: Clinical descriptions and diagnostic guidelines. Geneva, World Health Organization, 1992.

ZILBERGELD, B., ELLISON, C. R. Desire Discrepancies and Arousal Problems in Sex Therapy. Chapter in Principles and Practice of Sex Therapy. New York: Guilford Press, 1980. 